\title{
Mast- und Schlachtleistung bei Bullen und Färsen von Fleisch- rinderrassen und der Kreuzung Deutsch Angus x Fleckvieh
}

\begin{abstract}
Title of the paper: Fattening- and slaughter performance of bulls and heifers of beef breeds and the cross breed German Angus x Simmental

The objective of this study was to analyze the differences between fattening and slaughter performances as well as their compositions of meat cuts depending on breed and category of 158 cattle belonging to the breeds of German Angus (DA), Simmental (FV), Limousin (LIM) and the crossbreed DA X FV.

The Simmentals had the highest birth weight with $40.5 \mathrm{~kg}$ and the heaviest slaughter weight with $578 \mathrm{~kg}$. Although having the longest fattening time, Limousins had the lowest slaughter weight, but were able to compensate this disadvantage through the highest slaughter yield of $60 \%$ and with the biggest share of the pistol cut of $45 \%$. The German Angus compared with the other breeds appeared to have the most unfavourable results of performance, but the crossbreed (DA x FV) had the shortest fattening period, in which similar slaughter weights and weights of the two carcass halves were obtained. Both genotypes had a higher grade of adiposity and a visually lower muscularity, resulting in significantly lower shares of pistol cut and valuable meat cuts.

Heifers absolutely and relatively differ in terms of meat cuts compared with bulls. Only the share of valuable meat cuts was similar. Certainly bulls had a stronger accentuation of the forequarter. Shares of shoulder and neck were higher. However filet, roast beef and round shares of heifers were significantly higher.
\end{abstract}

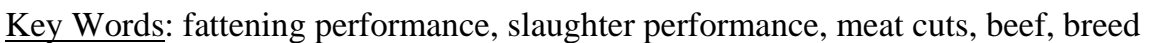

\section{Zusammenfassung}

Ziel dieser Untersuchung war es, anhand der Untersuchungsergebnisse von 158 Rindern der Rassen Deutsch Angus (DA), Fleckvieh (FV), Limousin (LIM) und der Kreuzung DA x FV rasse- und kategoriebedingte Unterschiede der Mast- und Schlachtleistung sowie deren Teilstückzusammensetzung zu analysieren.

Fleckvieh weist mit 40,5 kg das höchste Geburtsgewicht und mit 578 kg das schwerste Schlachtgewicht auf. Die Limousin Rinder hatten trotz einer längeren Mast die leichtesten Schlachtgewichte, können diese Nachteile aber durch die höchste mittlere Schlachtausbeute von $60 \%$ und dem größten Pistolenanteil von $45 \%$ wieder kompensieren. Bei Deutsch Angus lagen im Vergleich die ungünstigsten Ergebnisse vor, allerdings bestachen die Kreuzungstiere (DA x FV) durch eine verkürzte Mastzeit, in der vergleichbare Schlacht- und Zweihälftengewichte erreicht wurden. Beide Genotypen waren am stärksten verfettet und weniger stark in der Bemuskelung ausgeprägt, was auf einen signifikant geringeren Anteil der Pistole und der wertvollen Teilstücke zurückzuführen ist.

Färsen unterscheiden sich hinsichtlich der Teilstücke absolut und relativ signifikant von den Bullen. Einzig der Anteil wertvoller Teilstücke ist identisch. Allerdings weisen die Bullen eine stärkere Betonung des Vorderviertels auf. Hier waren die Anteile des Bugs und des Kamms höher. Filet-, Roastbeef- und Keulenanteil waren hingegen bei den Färsen signifikant höher.

Schlüsselwörter: Mastleistung, Schlachtleistung, Anteil wertvoller Teilstücke, Fleischrinderrassen

\section{1. $\quad$ Einleitung}

Die Mast- und Schlachtleistung von Mastrindern sind für den Landwirt eine wichtige Entscheidungsgrundlage sowohl für die zu wählende Genetik als auch für das geeignete Haltungsverfahren. Angestrebt werden hohe Geburts- und Mastendgewichte (GOLZE, 2001; MOLONEY und DRENNAN, 2006) bei niedrigem Schlachtalter, die 
mit hohen Netto- und Lebenstagszunahmen einhergehen und eine günstige Futterverwertung bei möglichst geringen Tierverlusten aufweisen (ZUPP et al., 1994; DANNENBERGER et al., 2006). Vom Schlachtbetrieb werden gesunde Schlachtrinder in möglichst homogenen Partien mit hohen Zweihälftengewichten, hohen Schlachtausbeuten und geringen Kühlverlusten erwartet (KALLWEIT et al., 1982; AUGUSTINI, 2000; LINK et al., 2003). Die Bezahlung der Schlachtrinder in deutschen Schlachthöfen basiert auf Fleischigkeit beziehungsweise dem Verfettungsgrad und weniger auf der Fleischqualität. Die erwünschten Einstufungen sind die Fleischigkeitsklassen E und U bzw. die Fettgewebeklassen 2 und 3. Dies begünstigt den Einsatz von großrahmigen und speziellen Mastrassen für die Fleischproduktion (FRELICH et al., 1998; KÖGEL et al., 2000; GOLZE, 2001; CHAMBAZ et al., 2001a, b; REICHARDT et al., 2002; JACUBEC et al., 2003). Weitere Fragen der Fleischrinderhaltung ist der Kostenaspekt und die hohen Qualitätsansprüche der Verbraucher (ENDER et al., 2000; CHAMBAZ et al., 2001a, b; REICHARDT et al., 2002; SURBT et al., 2006). Außerdem wird die Verbraucherakzeptanz von Rindfleisch durch artgerechte Haltungsverfahren (Mutterkuhhaltung) und den daraus resultierenden Fleischqualitäten beeinflusst (WEIHER und ZUPP, 1997; GOLZE, 2001).

Ziel der vorliegenden Untersuchung war die Prüfung des Rasse- bzw. Geschlechtseinflusses auf die Mast- und Schlachtleistung sowie auf die Teilstückzusammensetzung von Mastrindern aus der Mutterkuhhaltung.

2. Material und Methode

In der Mutterkuhherde der Sächsischen Landesanstalt für Tierzucht, Fischerei und Grünland in Köllitzsch wurden die Rassen Deutsch Angus (DA), Fleckvieh (FV), Limousin (LIM) und die Kreuzung DA x FV gehalten (s. Tab. 1). Die Kühe kalbten im Dezember und Januar ab und wurden ab Mitte April bis Mitte November mit ihren Kälbern auf der Weide gehalten. Bei Weideabtrieb wurden die Bullen- und Kuhkälber von ihren Müttern getrennt und in einem Tieflaufstall bis zur Schlachtreife gehalten. Die Bullen und Färsen erhielten Gras- und Maissilage ad libitum sowie 2 Kilogramm Kraftfutter. Alle Reinzucht und Kreuzungstiere wurden über zwei Weideperioden zeitgleich gehalten.

Tabelle 1

Verteilung der untersuchten Rinder auf Rasse und Kategorie $\left(\mathrm{n}_{\text {ges }}=158\right)$ (Frequency of the animals depending on breed and category $\left(\mathrm{n}_{\text {total }}=158\right)$ )

\begin{tabular}{lcc}
\hline & & Kategorie \\
Rasse & Bulle & Färse \\
\hline Deutsch Angus (DA) & 37 & 17 \\
Fleckvieh (FV) & 28 & 16 \\
Limousin (LIM) & 25 & 8 \\
DA x FV & 14 & 13 \\
\hline
\end{tabular}

Von 158 Rindern standen Geburts- und Schlachtgewicht, das Schlachtalter und daraus resultierend die Lebendtagszunahme (LTZ) zur Verfügung. Die Mastrinder wurden im Schlachthof der Firma Färber in Oschatz geschlachtet und nach DLG-Schnittführung (DLG, 1984) zerlegt. Dabei erfaßte man das Zweihälftengewicht (warm und kalt) sowie die Gewichte der einzelnen Teilstücke. Mit den vorliegenden Daten konnten 
Schlachtausbeute, Nettozunahme, Kühlverlust und der Anteil wertvoller Teilstücke berechnet werden. Darüber hinaus lagen die Klassifizierungsergebnisse für Fleischigkeit und Fettgewebe vor.

Die Daten wurden mit der PC-Version des Programmpaketes SAS (Statistic Analysis System, 1990) nach der Methode der kleinsten Quadrate unter Berücksichtigung der fixen Effekte Rasse und Kategorie statistisch analysiert. Die Signifikanzen zwischen den LSQ-Mittelwerten wurde mit Hilfe eines multiplen t-Testes geprüft. Folgendes varianzanalytisches Modell wurde den Berechnungen zugrunde gelegt:

$\begin{array}{lll}\mathrm{Y}_{\mathrm{ijk}}=\mu & +\mathrm{R}_{\mathrm{i}}+\mathrm{K}_{\mathrm{j}}+\mathrm{R}_{\mathrm{i}} \mathrm{x} \mathrm{K}_{\mathrm{j}}+\mathrm{e}_{\mathrm{ijk}} \\ \mathrm{Y}_{\mathrm{ijk}}= & \text { Beobachtungswert des Tieres } \\ \mu & = & \text { Mittelwert } \\ \mathrm{R}_{\mathrm{i}}= & \text { Fixer Effekt der i-ten Rasse } \\ \mathrm{K}_{\mathrm{j}}= & \text { Fixer Effekt der j-ten Kategorie } \\ \mathrm{RK}_{\mathrm{ij}}= & \text { Interaktion zwischen i-ter Rasse und j-ter Kategorie } \\ \mathrm{e}_{\mathrm{ijk}} & = & \text { zufälliger Restfehler der Beobachtung }\end{array}$

3. $\quad$ Ergebnisse und Diskussion

Mast- und Schlachtleistungen

Die Tabelle 2 zeigt, dass sich die einzelnen Genotypen hinsichtlich des Geburtsgewichtes signifikant unterscheiden. Zwar hatte die FV Gruppe die höchsten Geburtsund Schlachtgewichte, jedoch erreichten die Kreuzungstiere mit den geringsten Geburtsgewichten die signifikant größten Lebenstagszunahmen und relativ hohe Schlachtgewichte. In diesem Fall wird die Auffassung widerlegt, dass hohe Geburtsgewichte in positiver Beziehung zu Schlachtgewichten stehen (MOLONEY und DRENNAN, 2006).

Tabelle 2

LSQ-Mittelwerte (LM) und Standardfehler (SE) der Mast- und Schlachtleistungsmerkmale in Abhängigkeit von der Rasse (LSQ-means (LM) and standard error (SE) of the fattening performance and slaughter traits depending on breed)

\begin{tabular}{|c|c|c|c|c|c|c|c|c|}
\hline \multirow{3}{*}{ Merkmal } & \multicolumn{8}{|c|}{ Genotyp } \\
\hline & \multicolumn{2}{|c|}{ DA } & \multicolumn{2}{|c|}{ FV } & \multicolumn{2}{|c|}{ LIM } & \multicolumn{2}{|c|}{ DA $x F V$} \\
\hline & LM & SE & LM & SE & LM & SE & LM & SE \\
\hline Geburtsgewicht, kg & $38,6^{b}$ & 0,59 & $40,5^{\mathrm{a}}$ & 0,64 & $39,0^{\mathrm{ab}}$ & 0,82 & $38,2^{b}$ & 0,78 \\
\hline Schlachtgewicht, kg & $532^{\mathrm{ab}}$ & 10,0 & $578^{a}$ & 10,7 & $509^{b}$ & 13,9 & $548^{a b}$ & 13,2 \\
\hline Schlachtalter, d & $506^{\mathrm{a}}$ & 12,1 & $501^{\mathrm{a}}$ & 12,9 & $531^{a}$ & 16,8 & $436^{b}$ & 15,9 \\
\hline LTZ, g & $998^{\mathrm{c}}$ & 18,9 & $1106^{\mathrm{b}}$ & 20,6 & $906^{\mathrm{d}}$ & 26,2 & $1176^{\mathrm{a}}$ & 24,8 \\
\hline Schlachtausbeute, \% & $55,3^{b}$ & 0,35 & $54,2^{c}$ & 0,37 & $60,0^{\mathrm{a}}$ & 0,48 & $54,2^{c}$ & 0,46 \\
\hline Nettozunahme, g & $594^{b}$ & 10,9 & $644^{\mathrm{a}}$ & 11,6 & $586^{\mathrm{b}}$ & 15,1 & $684^{\mathrm{a}}$ & 14,3 \\
\hline Handelsklasse & $3,1^{\mathrm{a}}$ & 0,07 & $2,9^{b}$ & 0,08 & $2,6^{\mathrm{c}}$ & 0,10 & $3,2^{\mathrm{a}}$ & 0,09 \\
\hline Fettstufe & $3,3^{a}$ & 0,08 & $2,8^{b}$ & 0,08 & $2,4^{c}$ & 0,11 & $3,1^{a}$ & 0,10 \\
\hline
\end{tabular}

Ebenso wie FRELICH et al. (1989) und GOLZE (2001) fanden sich im Vergleich mit DA oder LIM in vorliegenden Untersuchungen die besten Schlachtgewichte und Lebenstagszunahmen bei den reinrassigen Fleckvieh bzw. Fleckviehkreuzungstieren. Das galt auch für den Vergleich der Nettozunahmen. Bei der Schlachtausbeute erreichte die LIM Gruppe mit 60,0 \% gefolgt von DA mit 55,3 \% die signifikant besten Ergebnisse. Diese auf hohe Fleischleistung gezüchtete Rasse französischen Ursprungs 
wird daher weit verbreitet in Reinzucht oder Kreuzung eingesetzt und zeigt sowohl bei den vorliegenden Untersuchungen als auch im Schrifttum (FRELICH et al., 1998; CHAMBAZ et al., 2001a, b; GOLZE, 2001) gute Ergebnisse. Bestätigt wird diese Aussage durch die Klassifizierung in Fleischigkeitsklassen, bei welcher die LIM Tiere mit ihrer sehr guten Bemuskelung die besten Werte aufwiesen. In der Fettgewebsklasse zeigen die DA Tiere die stärkste Verfettung bei geringster Bemuskelung. Ökonomisch gesehen sind diese Schlachtkörper bei den derzeit bestehenden Bezahlungspraktiken weniger interessant. Allerdings zeigen Untersuchungen von LINK et al. (2003), dass diese Tiere ein hohes Potential für eine gute Fleischqualität besitzen. Nach AUGUSTINI (2000) und ENDER et al. (2000) ist dies u.a. auf intensive Einlagerungen von intramuskulärem Fett bei dieser Rasse zurückzuführen. Bei DA Kreuzungstieren zeigte sich die gleiche Tendenz. Jedoch wurde dieser Nachteil durch die signifikant besseren Zuwachsleistungen kompensiert. So erreichten diese Tiere im Vergleich zu allen geprüften Genotypen die höchsten Lebenstags- und Nettozunahmen. Dieser Leistungsunterschied in den Wachstumsmerkmalen ist auf den Kreuzungseffekt zurückzuführen, was auch andere Autoren nachweisen konnten (FRELICH et al., 1998; KÖGEL, 2000; GOLZE, 2001).

Beim Vergleich der Tiere nach Kategorien (Tab. 3) ergaben sich erwartungsgemäß (ENDER und AUGUSTINI, 1998) bei allen Merkmalen signifikante Unterschiede zwischen den Ergebnissen der Bullen zu den Färsen.

Tabelle 3

LSQ-Mittelwerte (LM) und Standardfehler (SE) der Mast- und Schlachtleistungsmerkmale in Abhängigkeit von der Kategorie (LSQ-means (LM) and standard error (SE) of the fattening performance and slaughter traits depending on category)

\begin{tabular}{|c|c|c|c|c|}
\hline \multirow{3}{*}{ Merkmal } & \multicolumn{4}{|c|}{ Kategorie } \\
\hline & \multicolumn{2}{|c|}{ Bulle } & \multicolumn{2}{|c|}{ Färse } \\
\hline & LM & SE & LM & SE \\
\hline Geburtsgewicht, kg & $39,9^{b}$ & 0,42 & $38,3^{a}$ & 0,58 \\
\hline Schlachtgewicht, kg & $618^{\mathrm{b}}$ & 7,1 & $466^{\mathrm{a}}$ & 9,7 \\
\hline Schlachtalter, d & 481 & 9,6 & 506 & 11,7 \\
\hline LTZ, g & $1213^{b}$ & 13,5 & $880^{\mathrm{a}}$ & 18,5 \\
\hline Schlachtausbeute, \% & $57,1^{\mathrm{b}}$ & 0,23 & $54,8^{a}$ & 0,34 \\
\hline Nettozunahme, g & $737^{\mathrm{b}}$ & 7,7 & $517^{\mathrm{a}}$ & 10,5 \\
\hline Handelsklasse & $2,6^{\mathrm{b}}$ & 0,05 & $3,2^{a}$ & 0,07 \\
\hline Fettstufe & $2,7^{b}$ & 0,06 & $3,3^{\mathrm{a}}$ & 0,08 \\
\hline
\end{tabular}

So unterschieden sich die Bullen bereits durch ein höheres Geburtsgewicht von 1,6 kg. Dieser geschlechterbedingte Einfluss setzte sich bei den Wachstumsmerkmalen fort (GOYACHE et al., 2003; MOLONEY und DRENNAN, 2006) und äußerte sich auch durch bessere Ergebnisse der Bullen bei der Schlachtausbeute, den Fleischigkeits- und Fettgewebeklassen und bestätigt diesbezügliche Aussagen von ENDER und AUGUSTINI (1998).

Gewichte und Anteile der Teilstücke

Die Tabelle 4 zeigt die Teilstückgewichte sowie deren Anteile bei den einzelnen Genotypen. FV und LIM erreichten die höchsten, die Rasse DA die niedrigsten Zweihälftengewichte und -anteile. 
Tabelle 4

LSQ-Mittelwerte (LM) und Standardfehler (SE) der Teilstückgewichte und -anteile in Abhängigkeit von der Rasse (LSQ-means (LM) and standard error (SE) of the different meat cuts depending on breed)

\begin{tabular}{|c|c|c|c|c|c|c|c|c|}
\hline \multirow{3}{*}{ Merkmal } & \multicolumn{8}{|c|}{ Genotyp } \\
\hline & \multicolumn{2}{|c|}{ DA } & \multicolumn{2}{|c|}{ FV } & \multicolumn{2}{|c|}{ LIM } & \multicolumn{2}{|c|}{ DA x FV } \\
\hline & LM & $\mathrm{SE}$ & LM & $\mathrm{SE}$ & LM & SE & LM & $\mathrm{SE}$ \\
\hline Kühlverlust, \% & $1,54^{\mathrm{ab}}$ & 0,11 & $1,68^{\mathrm{a}}$ & 0,12 & $1,46^{\mathrm{ab}}$ & 0,16 & $1,29^{b}$ & 0,15 \\
\hline $\begin{array}{l}\text { Zweihälftengewicht } \\
\text { (kalt), kg }\end{array}$ & $290^{\mathrm{b}}$ & 5,7 & $310^{\mathrm{a}}$ & 6,1 & $300^{\mathrm{a}}$ & 7,9 & $295^{a b}$ & 7,5 \\
\hline $\begin{array}{l}\text { Anteil wertvoller } \\
\text { Teilstücke, kg }\end{array}$ & $172^{\mathrm{b}}$ & 3,3 & $187^{\mathrm{a}}$ & 3,5 & $187^{\mathrm{a}}$ & 4,5 & $173^{b}$ & 4,3 \\
\hline $\begin{array}{l}\text { Anteil wertvoller } \\
\text { Teilstücke, \% }\end{array}$ & $59,2^{c}$ & 0,20 & $60,5^{b}$ & 0,21 & $62,2^{a}$ & 2,27 & $58,8^{c}$ & 0,26 \\
\hline Pistole, kg & $121,2^{b}$ & 1,1 & $134,6^{\mathrm{a}}$ & 1,1 & $136,2^{\mathrm{a}}$ & 1,5 & $121,2^{b}$ & 1,4 \\
\hline Pistole, \% & $42,1^{\mathrm{c}}$ & 0,21 & $43,6^{b}$ & 0,22 & $45,5^{\mathrm{a}}$ & 0,29 & $41,3^{d}$ & 0,27 \\
\hline Keule, kg & $39,4^{b}$ & 0,72 & $44,4^{\mathrm{a}}$ & 0,77 & $45,5^{a}$ & 1,00 & $39,8^{b}$ & 0,95 \\
\hline Keule, \% & $27,4^{c}$ & 0,15 & $28,8^{b}$ & 0,16 & $30,4^{a}$ & 0,20 & $27,1^{\mathrm{c}}$ & 0,19 \\
\hline Roastbeef, kg & 11,5 & 0,23 & 12,1 & 0,25 & 12,0 & 0,32 & 11,5 & 0,31 \\
\hline Roastbeef, \% & 8,0 & 0,11 & 7,9 & 0,12 & 8,0 & 0,15 & 7,9 & 0,14 \\
\hline Filet, kg & $2,8^{b}$ & 0,07 & $3,2^{\mathrm{a}}$ & 0,07 & $3,3^{a}$ & 0,09 & $2,8^{b}$ & 0,09 \\
\hline Filet, \% & $1,9^{\mathrm{c}}$ & 0,02 & $2,1^{b}$ & 0,02 & $2,2^{\mathrm{a}}$ & 0,03 & $1,9^{\mathrm{c}}$ & 0,03 \\
\hline Bug, kg & $18,3^{b}$ & 0,39 & $19,4^{\mathrm{a}}$ & 0,42 & $19,3^{\mathrm{a}}$ & 0,54 & $18,2^{\mathrm{ab}}$ & 0,51 \\
\hline Bug, \% & $12,5^{\mathrm{ab}}$ & 0,08 & $12,4^{\mathrm{b}}$ & 0,090 & $12,8^{\mathrm{a}}$ & 0,11 & $12,3^{b}$ & 0,11 \\
\hline Kamm, kg & 13,8 & 0,36 & 14,5 & 0,38 & 13,5 & 0,49 & 14,2 & 0,47 \\
\hline Kamm, \% & $9,4^{\mathrm{a}}$ & 0,10 & $9,3^{a}$ & 0,11 & $8,9^{b}$ & 0,14 & $9,5^{\mathrm{a}}$ & 0,13 \\
\hline
\end{tabular}

Mit unterschiedlichen Buchstaben gekennzeichnete LSQ - Mittelwerte sind signifikant voneinander verschieden $(\mathrm{p}<0,05)$

Lag das Schlachtgewicht der FV Tiere noch erheblich über den LIM Tieren, so ist, bedingt durch die hohe Schlachtausbeute der LIM Tiere, dieser Rassenunterschied bei den Teilstückgewichten nicht mehr erkennbar, was auch DUFFEY (2000) nachweisen konnte. Der gleiche signifikante Rassenunterschied ergab sich bei den für die Vermarktung wichtigen Merkmalen Pistole und Anteil wertvoller Teilstücke, sowie den Einzelteilstücken Keule, Filet und Bug. Die Teilstückgewichte und deren Anteile für die Kategorien Bulle und Färse sind in Tabelle 5 dargestellt.

Tabelle 5

LSQ-Mittelwerte (LM) und Standardfehler (SE) der Teilstückgewichte und -anteile in Abhängigkeit von der Kategorie (LSQ-means (LM) and standard error (SE) of the different meat cuts depending on category)

\begin{tabular}{|c|c|c|c|c|}
\hline \multirow{3}{*}{ Merkmal } & \multicolumn{4}{|c|}{ Kategorie } \\
\hline & \multicolumn{2}{|c|}{ Bulle } & \multicolumn{2}{|c|}{ Färse } \\
\hline & LM & SE & LM & SE \\
\hline Kühlverlust, \% & $1,79^{\mathrm{a}}$ & 0,08 & $1,2^{b}$ & 0,11 \\
\hline Zweihälftengewicht (kalt), kg & $347^{\mathrm{a}}$ & 4,0 & $251^{b}$ & 5,5 \\
\hline Anteil wertvoller Teilstücke, kg & $208^{a}$ & 2,3 & $151^{\mathrm{b}}$ & 3,2 \\
\hline Anteil wertvoller Teilstücke, \% & 60,2 & 0,14 & 60,2 & 0,19 \\
\hline Pistole, $\mathrm{kg}$ & $72,9^{\mathrm{a}}$ & 0,76 & $55,4^{b}$ & 1,04 \\
\hline Pistole, \% & $42,2^{\mathrm{a}}$ & 0,15 & $44,1^{\mathrm{b}}$ & 0,20 \\
\hline Keule, kg & $48,1^{\mathrm{a}}$ & 0,52 & $36,4^{b}$ & 0,70 \\
\hline Keule, \% & $27,8^{\mathrm{a}}$ & 0,10 & $29,0^{b}$ & 0,14 \\
\hline Roastbeef, kg & $13,4^{\mathrm{a}}$ & 0,17 & $10,2^{b}$ & 0,23 \\
\hline Roastbeef, \% & $7,8^{\mathrm{a}}$ & 0,08 & $8,1^{\mathrm{b}}$ & 0,11 \\
\hline Filet, kg & $3,4^{\mathrm{a}}$ & 0,05 & $2,6^{\mathrm{b}}$ & 0,06 \\
\hline Filet, \% & $2,0^{\mathrm{a}}$ & 0,01 & $2,1^{\mathrm{b}}$ & 0,02 \\
\hline Bug, kg & $22,4^{\mathrm{a}}$ & 0,28 & $15,2^{b}$ & 0,38 \\
\hline Bug, \% & $2,9^{\mathrm{a}}$ & 0,06 & $2,1^{\mathrm{b}}$ & 0,08 \\
\hline Kamm, kg & $16,8^{\mathrm{a}}$ & 0,25 & $11,2^{b}$ & 0,34 \\
\hline Kamm, \% & $9,7^{\mathrm{a}}$ & 0,07 & $8,9^{b}$ & 0,10 \\
\hline
\end{tabular}

Mit unterschiedlichen Buchstaben gekennzeichnete LSQ - Mittelwerte sind signifikant voneinander verschieden $(\mathrm{p}<0,05)$ 
Außer den um 0,8 \% höheren Kühlverlust, welcher durch den geringeren Verfettungsgrad hervorgerufen wird, erreichten die Bullen erwartungsgemäß die bessere Fleischfülle in Form höherer Teilstückgewichte (KALLWEIT et al., 1988; ENDER und AUGUSTINI, 1998). Ausdruck dieses geschlechtbedingten Unterschiedes ist bei großen Differenzen im Gesamtgewicht wertvoller Teilstücke, ein gleich hoher prozentualer Anteil dieser Teilstücke in beiden Kategorien. Bei den Bullen fand sich ein höherer Anteil an Bug und Kamm während die Färsen diesen bei den Merkmalen Keule, Roastbeef und Filet erreichten.

Im Ergebnis dieser Untersuchung läßt sich schlußfolgern, dass die FV Tiere sowohl aus der Sicht der Wachstumsleistung als auch der Vermarktung die relativ besten Ergebnisse aufwiesen. Die LIM Gruppe war zwar hinsichtlich der Wachstumsleistung den FV unterlegen, kompensierte aber diese Minderleistung durch das sehr gute Ausschlachtergebnis, was seinen besonderen Ausdruck im höchsten Pistolenanteil fand. Die DA Gruppe erwies sich in vielen der untersuchten Positionen als die ungünstigste Variante. Besonders hervorzuheben ist die mit Abstand beste Zunahmeleistung der DA $\mathrm{x}$ FV Kreuzungstiere. Sowohl die DA als auch die Kreuzungstiere wiesen die ungünstigsten Ergebnisse bei den Fleischigkeits- und Fettgewebeklassen auf und hatten damit die schlechtesten Vermarktungsergebnisse.

Beim Vergleich der Bullen mit den Färsen, die in vorliegender Untersuchung beide ein Schlachtalter von ca. 500 Tagen aufwiesen, erreichten die Bullen erwartungsgemäß die absolut höchsten Gewichte. Eine Färsenmast der hier geprüften Genotypen erwies sich aus Sicht des Vermarktungsergebnisses als weniger erfolgreich. Die in einigen Fleischteilstückpositionen guten Ergebnisse der Färsen hatten allerdings eine größere Verfettung und damit schlechtere Handelsklasseneinstufungen zur Folge und stellen das hohe Schlachtalter dieser Gruppe in Frage. Das in vorliegenden Untersuchungen erreichte Ergebnis lässt bei intensiver Mast weibliche Rinder aus Fleischrinderrassen oder deren Gebrauchskreuzungen bei kürzerer Mastzeit (GERHARDY und GRIEPENKERL, 1994; SCHEEDER et al., 1996) vermutlich ein besseres Ergebnis, auch bei einer dann erfolgenden Einstufung der Tiere in die Kategorie Jungrindfleisch (JR), erwarten.

AUGUSTINI, C.:

\section{Literatur}

Fleisch im Umfeld von Ökologie und Nachhaltigkeit. Kulmbacher Reihe Bd. 17, Institut für Fleischerzeugung und Vermarktung der Bundesanstalt für Fleischforschung, Kulmbach (2000)

CHAMBAZ, A.; MOREL, I.; SCHEEDER, M.R.L.; KREUZER, M.; DUFEY, P.-A.:

Characteristics of steers of six beef fattened from eight month of age and slaughtered at a level of intramuscular fat. I: Growth performance and carcass quality. Arch. Tierz., Dummerstorf 44 (2001a), 395-411

CHAMBAZ, A.; KREUZER, M.; SCHEEDER, M.R.L.; DUFEY, P.-A.:

Characteristics of steers of six beef fattened from eight month of age and slaughtered at a level of intramuscular fat. II: Meat quality. Arch. Tierz., Dummerstorf 44 (2001b), 473-488

DANNENBERGER, D.; NUERNBERG, K.; NUERNBERG, G.; ENDER, K.:

Carcass- and meat quality of pasture vs concentrate fed German Simmental and German Holstein bulls. Arch. Tierz., Dummerstorf 49 (2006), 315-328

DLG:

DLG Schnittführung für die Zerlegung der Schlachtkörper von Rind, Kalb, Schwein und Schaf. Deutsche Landwirtschaftsgesellschaft, Frankfurt/M (1984)

DUFFY; P.-A.; CHAMBAZ, A.; MOREL, I.; CHASSOT, A.:

Mastleistung von Ochsen sechs verschiedener Fleischrassen. Eidgenössische Forschungsanstalt für

Nutztiere (RAP), Posieux (2002), http://www.sar.admin.ch/zs/afo

ENDER, K.; AUGUSTINI, C.: 
Schlachttierwert von Rind und Kalb in: BRANSCHEID, W.; HONIKEL, K.O.; LENGERKEN von, G.; DROCHNER, W.; TROEGER, K.: Qualität von Fleisch und Fleischwaren, Bd. 1, Deutscher Fachverlag Frankfurt/M (1998)

ENDER, K.; NÜRNBERG, K.; ENDER, B.:

Rindfleisch - Fleisch hoher ernährungsphysiologischer Wertigkeit. Arch. Tierz., Dummerstorf 43 (2000) Sonderheft, 84-90

FRELICH, J.; VORISKOVA, A.; KUNIK, J.; KVAPILIK, J.:

Mast- und Schlachtleistungen von Bullen aus Gebrauchskreuzungen tschechischer Rinderrassen mit Fleischrinderrassen. Arch. Tierz., Dummerstorf 41 (1998), 533-544

GERHARDY, H.; GRIEPENKERL, L.: Cereal beef production from crossbred heifers. Arch. Tierz., Dummerstorf 37 (1994), 481-492

GOLZE, M.:

Wachstum, Schlachtleistung und Schlachtkörperqualität von Weidemastkälbern aus der Mutterkuhhaltung mit Fleischrindrassen. Arch. Tierz., Dummerstorf 44 (2001), 621-627

GOYACHE, F.; FERNANDEZ, I.; ROYO, L.; ALVAREZ, I. ; GUTIERREZ, L.P.:

Factors affecting actual weaning weight, preweaning avarage daily gain and relative growth rate in Asturian de los Valles beef cattle breed. Arch. Tierz., Dummerstorf 46 (2003), 235-243

JACUBEC, V.; SCHLOTE, W.; RIHA, J.; MAJZLIK, I.: Comparison of growth traits of eight beef cattle breeds in the Czech Republic. Arch. Tierz., Dummerstorf 46 (2003), 143-153

KALLWEIT, E.; FRIES, R.; KIELWEIN, G.; SCHOLTYSSEK, S.:

Qualität tierischer Nahrungsmittel, Vlg. Eugen Ulmer, Stuttgart (1988)

KÖGEL, J.; PICKL, M.; ROTT, J.; HOLLWICH, W.; SARREITER, R.; MEHLER, N.:

Kreuzungsversuch mit Charolais, Blond d' Aquitaine und Limousin auf Fleckviehkühe. Züchtungskunde 72 (2000), 201-216

LINK, G.; WILLEKE, H.; GOLZE, M.; BERGFELD, U.; SCHÖBERLEIN, L.:

Einfluss der Rasse und Kategorie auf die Fleischqualitätsmerkmale vor und nach der Reifung von Rindfleisch. Fleischwirtschaft (2003), 103-106

MOLONEY, A.; DRENNAN, M.:

The influence of calf birth weight on selected beef quality characteristics. Arch. Tierz., Dummerstorf 49 (2006) Special Issue, 68-71

REICHARDT, W.; WARZECHA, H.; GERNAND, E.; HARTUNG, H.; ECKERT, B.:

Erhebungen zum Hämpigmentgehalt, zu Reflexionswerten sowie zum Fettsäuremuster des intramuskulären Fettes vom Musculus longissimus dorsi Thüringer Rinder in Abhängigkeit von Mastform und Rassetyp. Arch. Tierz., Dummerstorf 45 (2002), 111-127

SAS:

SAS User’s Guide, Statistical Analysis System Institute, Inc, Cary, NC, USA (1990)

SCHEEDER, R.L.; GERHARDY, H.; LANGHOLZ, H.-J.:

Untersuchungen zur Verwertungseignung unterschiedlicher Muskeln weiblicher Jungmastrinder. Arch. Tierz., Dummerstorf 39 (1996), 415-429

SURBT, J.; FILIPCIK, R.; ZUPKA, Z.; FIALOVA, M.; DRACKOVA, E.:

The content of polyunsaturated fatty acids in intramuscular fat of beef cattle in different breeds and crossbreeds. Arch. Tierz., Dummerstorf 49 (2006), 340-350

WEIHER, O.; ZUPP, W.:

Stand und Perspektive der Mutterkuhhaltung. Arch. Tierz., Dummerstorf 40 (1997) Sonderheft, 55-61

ZUPP, W.; WEIHER, O.; MARTIN, J.:

Fleischrindzucht und Mutterkuhhaltung bei veränderten Rahmenbedingungen. Arch. Tierz., Dummerstorf 37 (1994) Sonderheft, 49-55

Eingegangen: 2006-05-17

Akzeptiert: 2007-04-05

Autor für Korrespondenz

Prof. Dr. HENNING WILLEKE

Fachhochschule Weihenstephan, Abteilung Triesdorf

D-91746 WEIDENBACH-TRIESDORF

E-Mail: henning.willeke@fh-weihenstephan.de 